\section{Plain X-ray and computed tomography of the orbit in cases and suspected cases of intraocular foreign body}

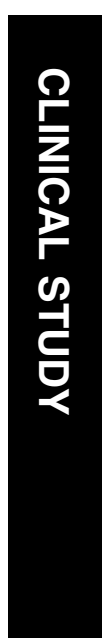
cases and suspected cases of IOFB.

Setting Royal Victoria Eye and Ear Hospital, Dublin, Ireland.

Results Plain X-rays were performed in the absence of clinically evident ocular penetration in $177(87 \%)$ cases, and no IOFB was demonstrated in any of these radiographs. Twenty-seven (13\%) plain $\mathrm{X}$-ray radiographs were obtained in the presence of clinically evident ocular penetration, and an IOFB was clinically visible in $19(70 \%)$ of these cases. CT scans were undertaken in $21(\mathbf{1 0 \%})$ of the 204 patients. Of these CT images, 9 (43\%) and $12(57 \%)$ were undertaken in the absence and presence of clinically evident ocular penetration, respectively. None $(0 \%)$ and all (100\%) of the CT scans obtained in the absence and presence of clinically evident ocular penetration demonstrated an IOFB, respectively.

Conclusion Plain X-ray and CT orbital imaging are non-contributory in the absence of clinically evident ocular penetration. In the presence of clinically evident ocular penetration, and where an IOFB is clinically visible, plain X-ray orbital radiography may have a role in excluding multiple IOFBs. In the presence of clinically evident ocular penetration, but where an IOFB is not clinically visible, CT orbital imaging remains the investigation of choice, and the role of
pre-CT plain X-ray orbital radiography, as recommended by the guidelines of the Royal College of Radiologists, merits re-evaluation. Eye (2008) 22, 1373-1377; doi:10.1038/sj.eye.6702876; published online 8 June 2007

Keywords: X-ray; computed tomography (CT) scan; orbit; intraocular foreign body (IOFB)

A Saeed ${ }^{1}$, L Cassidy ${ }^{2}$, DE Malone ${ }^{3}$ and S Beatty ${ }^{1}$

\section{Introduction}

X-ray orbital radiography (plain and computed tomography (CT) imaging) to detect and/or localize intraocular foreign bodies (IOFBs) is commonly performed on ophthalmic patients with a history of exposure to high-velocity particles. The booklet 'Making the Best Use of a Department of Clinical Radiology' issued by the Royal College of Radiologists (MBUR RCR) ${ }^{1}$ states that a single 'soft' lateral X-ray is the only projection required to exclude a metallic foreign body, and that images with eye movement are indicated only in those cases where the intraocular position of a radiologically proven suggest that CT orbital imaging in cases or suspected cases of IOFB should be undertaken only when the plain X-ray orbital radiograph fails to show a strongly suspected foreign body, which may not be metallic, when multiple foreign bodies are present, or when it remains uncertain whether a radiologically proven foreign body is intraocular. The MBUR RCR guidelines aim to limit patients' exposure to radiation and to reduce non-contributory yet costly examinations in the Accident and Emergency and/or Radiology Departments. foreign body is uncertain. These guidelines also
${ }^{1}$ Department of Ophthalmology, Waterford Regional Hospital, Waterford, Ireland

${ }^{2}$ Department of Ophthalmology, Royal Victoria Eye and Ear Hospital, Dublin, Ireland

${ }^{3}$ Department of Radiology, Royal Victoria Eye and Ear Hospital, Dublin, Ireland

Correspondence: A Saeed, Department of Ophthalmology, Waterford Regional Hospital, Dunmore Road, Waterford, Ireland Tel/Fax: + 35351848000 . E-mail: aymantalat@ gmail.com

Received: 22 January 2007 Accepted in revised form: 26 April 2007 Published online: 8 June 2007 
Bray and Griffith ${ }^{2}$ reported that plain X-rays of the orbits are unnecessary in patients exposed to high-velocity particles where there is no clinical evidence of ocular penetration, and suggested that plain X-ray orbital radiography should be restricted to those patients with clinical evidence of ocular penetration. They did not evaluate the role of CT orbital imaging in cases and suspected cases of IOFB.

The purpose of this study was to evaluate the roles of plain X-ray and CT orbital radiography in patients where an IOFB is present or suspected.

\section{Methods}

The records of all patients who underwent X-ray orbital radiography (plain films $\pm \mathrm{CT}$ imaging) between August 2001 and July 2002, where an IOFB was present or suspected, in the Radiology Department of the Royal Victoria Eye and Ear Hospital, Dublin, Ireland, were retrieved. The data recorded for each subject can be classed as demographic, clinical, radiological, and therapeutic.

A standard plain $\mathrm{X}$-ray orbital radiograph was taken according to a strict protocol, which included a non-screened lateral view, with occiptomental eye-moving images obtained only if the initial view was suggestive of an IOFB.

In compliance with the MBUR RCR guidelines, ${ }^{1}$ CT orbital imaging was undertaken only in cases or suspected cases of IOFB following a plain orbital X-ray radiograph. All scans were performed on a spiral CT scanner (Siemens Somatom) using a $3 \mathrm{~mm}$ slice thickness and a pitch of $1: 1$. All the images (plain $\mathrm{X}$-ray or CT) were reported by one of two consultant radiologists.

\section{Results}

Two hundred and ten patients with an IOFB or suspected IOFB underwent $X$-ray orbital radiography (plain $X$-ray \pm CT imaging) during the study period. Of these, the medical records were available in 204 cases (197 males and 7 females). The mean $( \pm S D)$ age was 39 $( \pm 16.8)$ years, with a range of 7-91 years.

The clinical details and radiological findings are given, for cases where plain X-ray orbital radiography and CT orbital imaging were undertaken, in Tables 1 and 2, respectively. In brief, of the $21 \mathrm{CT}$ orbital scans undertaken, $11(52.4 \%), 8(38.1 \%)$, and $2(9.5 \%)$ were performed in patients in whom an IOFB was seen, was not seen, and was indeterminate, respectively, on plain $\mathrm{X}$-ray orbital radiography. Sensitivity, specificity, and predictive values of plain X-ray and CT orbital radiography in cases and suspected cases of IOFB are given in Table $3 .^{3}$

\section{Discussion}

X-ray orbital radiography, whether plain X-ray or CT imaging, is commonly performed on ophthalmic patients with a history of exposure to high-velocity particles and a suspected or clinically visible IOFB. The sensitivity of plain orbital X-rays in these cases has been the subject of investigation in the past, with previous reports indicating that radiopaque foreign bodies are detected by plain $X$-ray radiography in 70-90\% of cases. ${ }^{2,4}$ Of note, failure of plain X-ray to detect an IOFB is a well-recognized phenomenon, and reflects the variable sizes, compositions, and locations of IOFBs. ${ }^{5-7}$

Interestingly, $87 \%$ of the plain orbital $\mathrm{X}$-rays undertaken in this study were performed in the absence of clinically evident ocular penetration, with a yield

Table 1 Clinical details and radiological findings in 204 cases and suspected cases of intraocular foreign body that underwent plain X-ray orbital radiography

\begin{tabular}{|c|c|c|c|c|}
\hline & \multicolumn{2}{|c|}{$\begin{array}{l}\text { Clinically evident ocular penetration } \\
\qquad \mathrm{n}=27 \text { (13\%) }\end{array}$} & \multicolumn{2}{|c|}{$\begin{array}{l}\text { No clinical evidence of ocular penetration } \\
\qquad \mathrm{n}=177 \text { ( } 87 \%)\end{array}$} \\
\hline & $\begin{array}{l}\text { IOFB clinically visible } \\
(\mathrm{n} ; \%)\end{array}$ & $\begin{array}{l}\text { IOFB not clinically } \\
\text { visible }(\mathrm{n} ; \%)\end{array}$ & $\begin{array}{l}\text { IOFB clinically } \\
\text { visible (n; \%) }\end{array}$ & $\begin{array}{l}\text { IOFB not clinically } \\
\text { visible }(\mathrm{n} ; \%)\end{array}$ \\
\hline IOFB evident on plain orbital X-rays & $18(95)$ & $7(87)^{a}$ & $0(0)$ & $1(0.6)^{b}$ \\
\hline No IOFB evident on plain orbital X-rays & $1(5)^{c}$ & $0(0)$ & $0(0)$ & $174(98.3)$ \\
\hline $\begin{array}{l}\text { Indeterminate with respect to the } \\
\text { presence of IOFB on plain orbital X-rays }\end{array}$ & $0(0)$ & $1(13)^{\mathrm{d}}$ & $0(0)$ & $2(1.1)^{\mathrm{e}}$ \\
\hline Total & $19(70)$ & $8(30)$ & $0(0)$ & $177(100)$ \\
\hline
\end{tabular}

IOFB, intraocular foreign body.

${ }^{a}$ Of these, one eye exhibited extensive ocular damage and underwent primary enucleation, and the remaining six went on to CT imaging of the orbit.

${ }^{b}$ False positive result, and it was deemed that no further radiological investigations or management were indicated.

${ }^{c}$ False negative (ie plain X-ray failed to identify a clinically visible metallic foreign body).

${ }^{\mathrm{d}}$ An IOFB was ultimately identified on a CT scan.

eNo further radiological investigation or management deemed necessary. 
Table 2 Clinical details and radiological findings in 21 cases and suspected cases of intraocular foreign body that underwent CT orbital imaging

\begin{tabular}{|c|c|c|c|c|}
\hline & \multicolumn{2}{|c|}{$\begin{array}{l}\text { Clinically evident ocular penetration } \\
\qquad \mathrm{n}=12(57 \%)\end{array}$} & \multicolumn{2}{|c|}{$\begin{array}{l}\text { No clinical evidence of ocular penetration } \\
\qquad \mathrm{n}=9(43 \%)\end{array}$} \\
\hline & $\begin{array}{l}\text { IOFB clinically } \\
\text { visible (n; \%) }\end{array}$ & $\begin{array}{l}\text { IOFB not clinically } \\
\text { visible }(\mathrm{n} ; \%)\end{array}$ & $\begin{array}{l}\text { IOFB clinically } \\
\text { visible }(\mathrm{n} ; \%)\end{array}$ & $\begin{array}{l}\text { IOFB not clinically } \\
\text { visible }(\mathrm{n} ; \%)\end{array}$ \\
\hline IOFB evident on orbital CT scan & $5(100)$ & $7(100)$ & $0(0)$ & $0(0)$ \\
\hline No IOFB evident on orbital CT scan & $0(0)$ & $0(0)$ & $0(0)$ & $9(100)$ \\
\hline $\begin{array}{l}\text { Indeterminate with respect to the } \\
\text { presence of IOFB on orbital CT scan }\end{array}$ & $0(0)$ & $0(0)$ & $0(0)$ & $0(0)$ \\
\hline Total & $5(42)$ & $7(58)$ & $0(0)$ & $9(100)$ \\
\hline
\end{tabular}

$\mathrm{CT}$, computed tomography; IOFB, intraocular foreign body.

Table 3 Sensitivity, specificity, and predictive values of plain $\mathrm{X}$-ray and CT orbital radiography in cases and suspected cases of IOFB

\begin{tabular}{lcc}
\hline & $\begin{array}{c}\text { Plain X-ray of the } \\
\text { orbits }\end{array}$ & $\begin{array}{c}\text { CT imaging of the } \\
\text { orbits }\end{array}$ \\
\hline Sample size & 204 & 21 \\
Prevalence of IOFB & $13 \%$ & $57 \%$ \\
Sensitivity (95\% CI) & $96 \%(88-100 \%)$ & $100 \%(100-100 \%)$ \\
Specificity (95\% CI) & $99 \%(98-100 \%)$ & $100 \%(100-100 \%)$ \\
PPV & $96 \%$ & $100 \%$ \\
NPV & $99 \%$ & $100 \%$ \\
\hline
\end{tabular}

CI, confidence interval; CT, computed tomography; IOFB, intraocular foreign body; NPV, negative predictive value; PPV, positive predictive value.

of $0(0 \%)$, consistent with an earlier report by Bray and Griffith. ${ }^{2}$ Of the 27 (13\%) plain X-ray orbital radiographs performed in patients with clinically evident ocular penetration following exposure to high-velocity particles, $19(70 \%)$ exhibited a clinically visible IOFB. In these cases, therefore, the clinical findings were simply confirmed by plain X-ray orbital radiography, and the contribution to the management of these cases by such imaging rests on its documentary value and on its ability to exclude multiple IOFBs. Furthermore, in eight cases of clinically confirmed ocular penetration following exposure to high-velocity particles, in the absence of a clinically visible IOFB, plain X-ray orbital radiography did furnish the clinician with radiological evidence of an IOFB in seven $(87 \%)$ cases.

Previous reports have shown that CT orbital imaging is superior to orbital plain X-ray orbital radiography for the detection and localization of IOFBs, although CT is associated with greater exposure to radiation than plain X-ray radiography. ${ }^{8-10}$ In contrast to plain X-ray orbital radiography, the majority $(12 ; 57 \%)$ of orbital CT scans in our study were performed on patients with clinically evident ocular penetration. However, five (42\%) of these cases had a clinically visible IOFB and radiological confirmation of the IOFB on plain X-rays of the orbit. In these cases, multiple foreign bodies were not evident on the plain X-ray orbital radiographs, and the value of CT images in this setting is therefore questionable, given that localization of the IOFB was achieved clinically.

Similar to plain X-ray orbital radiography, CT findings correlated well with clinical findings, in that all CT orbital images that were performed on eyes exposed to high-velocity particles with clinically evident ocular penetration, whether or not an IOFB was clinically visible, provided radiological evidence of an IOFB, whereas no CT scans performed in the absence of clinically evident ocular penetration following exposure to high-velocity particles showed radiological evidence of an IOFB.

The MBUR RCR guidelines stipulate that CT imaging should be undertaken only when the preceding plain X-ray orbital radiograph fails to show a strongly suspected foreign body, which may not be metallic, when multiple foreign bodies are present, or when it is not certain whether a foreign body already demonstrated is intraocular. ${ }^{1}$ Our experience suggests that, where there is clinical evidence of ocular penetration but an IOFB is not clinically visible, CT imaging would be necessary irrespective of the result of the preceding plain X-ray orbital radiograph. This is so for two reasons. First, CT imaging would identify an IOFB that might have gone undetected on orbital plain X-ray. Second, when an IOFB is demonstrated on the plain X-ray orbital radiograph, CT imaging would be necessary for its accurate localization.

It could be argued that plain X-ray orbital radiography before CT imaging acts as a guide to the radiologist whether 3 or $6 \mathrm{~mm}$ thick CT sections are to be taken. Six millimetre scans, which are associated with less radiation dosage than $3 \mathrm{~mm}$ scans, would be sufficient to detect and localize an IOFB that is visible on the plain X-ray orbital radiography. ${ }^{8}$ Modern CT scanners (multidetector CT scanning (MCT)) preclude the need for such measures because of their ability to obtain much thinner scans 


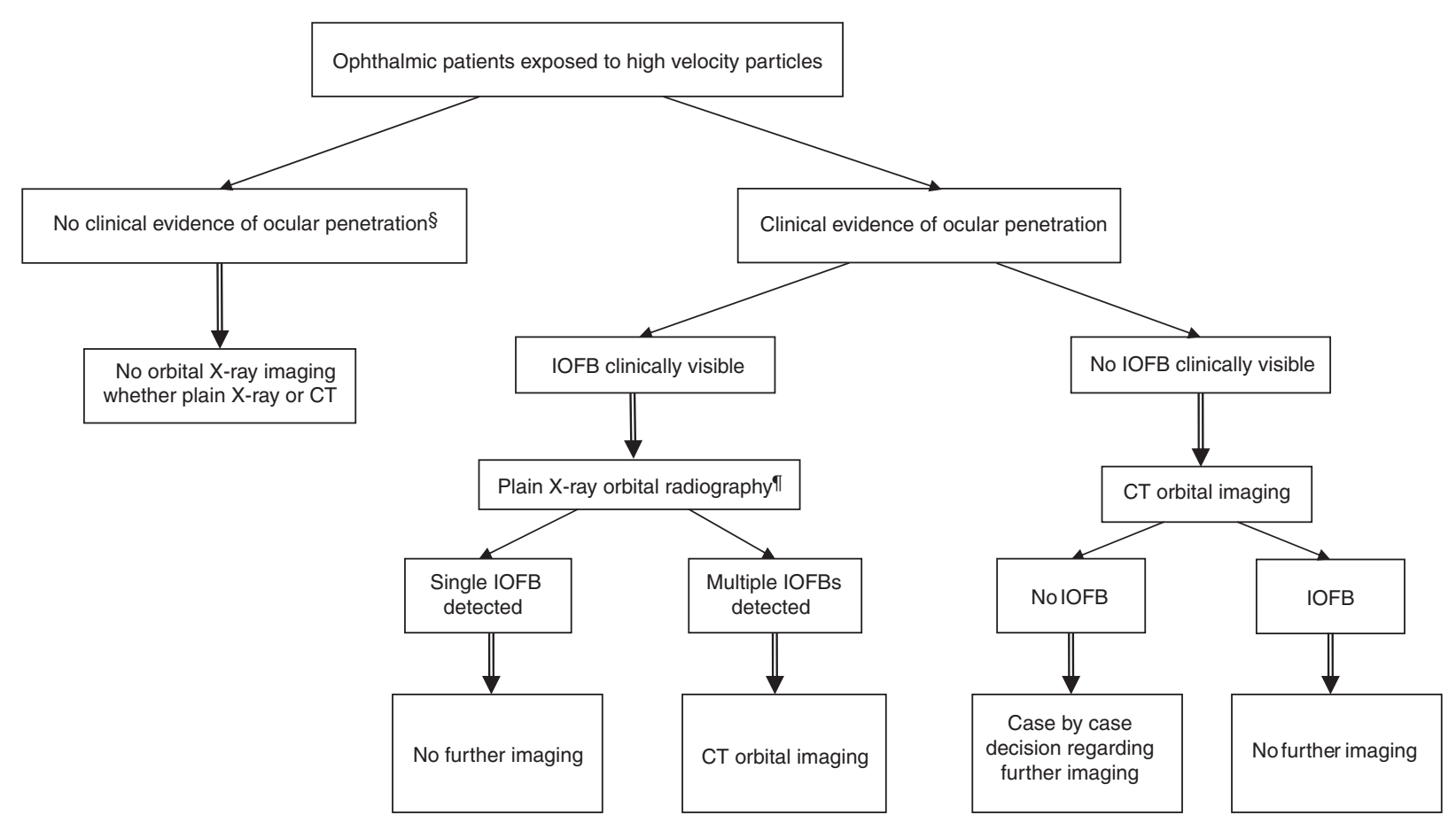

$\S$ : If a considerable amount of time has passed since exposure to high velocity particles, clinical evidence of ocular penetration (e.g. subconjunctival haemorrhage) may have resolved; I: The value of plain X-ray orbital radiography in the absence of a history of exposure to multiple IOFBs, such as blast injuries, is questionable

Figure 1 Flow diagram of the proposed X-ray (plain X-ray and / or CT imaging) orbital radiography pathway in cases and suspected cases of intraocular foreign body (IOFB).

(as thin as $1 \mathrm{~mm}$ ) with much less radiation exposure than conventional scans. ${ }^{11,12}$ The logistical burden of arranging pre-CT plain X-ray orbital radiography, and having such images reviewed, may adversely affect the speedy management of patients, especially in eye-dedicated units that may be remote from radiological services.

Conversely, where a metallic IOFB is clinically visible, plain X-ray orbital radiography without subsequent CT orbital imaging may serve to ensure that multiple IOFBs are not present. However, in the presence of a reliable history that excludes multiple IOFBs, the value of such imaging is questionable.

In conclusion, a thorough ophthalmic examination, with particular attention directed towards the likelihood of ocular penetration, and including gonioscopy (where appropriate) and detailed dilated fundoscopy remains the mainstay of management of ophthalmic patients exposed to high-velocity particles. The results of this series confirm other authors' conclusions ${ }^{2}$ that patients without clinical evidence of ocular penetration do not need to undergo orbital imaging of any kind. However, such a recommendation applies only to those patients who are exposed to high-velocity particles in the recent past, where clinical evidence of ocular penetration (eg subconjunctival haemorrhage) is not likely to have resolved. Where a metallic IOFB is clinically visible, plain X-ray orbital radiography without subsequent CT orbital imaging may be required to ensure that multiple IOFBs are not present, before proceeding to surgery (Figure 1). Given that in no case did plain X-ray of the orbit detect an IOFB that went undetected on subsequent CT imaging, and that the detection of an IOFB on plain X-ray radiography does not contribute to the decision-making process with respect to proceeding to CT imaging where there is a clinically evident ocular penetration but an IOFB is not clinically visible, it would appear that the standard imaging guidelines by the RCR need to be amended with respect to the stipulation that CT imaging must be preceded by plain X-ray orbital radiography. ${ }^{1}$ Finally, if a non-radiopaque IOFB is still strongly suspected following negative CT imaging, other imaging modalities such as ocular ultrasound or magnetic resonance imaging may need to be considered.,13,14

\section{References}

1 Royal College of Radiologists. Making the Best Use of a Department of Clinical Radiology. Guidelines for Doctors, 5th ed. London, 2003. 
2 Bray LC, Griffiths PG. The value of plain radiography in suspected intraocular foreign body. Eye 1991; 5: 751-754.

3 Maceneaney PM, Malone DE. The meaning of diagnostic test results: a spreadsheet for swift data analysis. Clin Radiol 2000; 55: 227-235.

4 Bryden FM, Pyott AA, Bailey M, McGhee CN. Real time ultrasound in the assessment of intraocular bodies. Eye 1990; 4: 727-731.

5 McElvanney AM, Fielder AR. Intraocular foreign body missed by radiography. BMJ 1993; 306: 1060-1061.

6 Barnes E, Griffiths M, Elliott A. Intraocular foreign body missed by computed tomography. BMJ 1993; 306: 1542.

7 DeAngelis D, Howcroft M, Aslanides I. Siderosis bulbi with an undetectable intraocular foreign body. Can J Ophthalmol 1999; 34: 341-342.

8 Etherington RJ, Hourihan MD. Localisation of intraocular and intraorbital foreign bodies using computed tomography. Clin Radiol 1989; 40: 610-614.

9 Zinreich SJ, Miller NR, Aguayo JB, Quinn C, Hadfield R, Rosenbaum AE. Computed tomography three-dimensional localization and compositional evaluation of intraocular and orbital foreign bodies. Arch Ophthalmol 1986; 104: 1477-1482.

10 Oikarinen KS, Nieminen TM, Makarainen H, Pyhtinen J Visibility of foreign bodies in soft tissue in plain radiographs, computed tomography, magnetic resonance imaging, and ultrasound. Int J Oral Maxillofac Surg 1993; 22: 119-124.

11 Dass AB, Ferrone PJ, Chu YR, Esposito M, Gray L. Sensitivity of spiral computed tomography scanning for detecting intraocular foreign bodies. Ophthalmology 2001; 108: 2326-2328.

12 Lakits A, Prokesch R, Scholda C, Nowotny R, Kaider A, Bankier A. Helical and conventional CT in the imaging of metallic foreign bodies in the orbit. Acta Ophthalmol Scand 2000; 78: 79-83.

13 Barash D, Goldenberg-Cohen N, Tzadok D, Lifshitz T, Yassur Y, Weinberger D. Ultrasound biomicroscopic detection of anterior ocular segment foreign body after trauma. Am J Ophthalmol 1998; 126: 197-202.

14 LoBue TD, Deutsch TA, Lobick J, Turner DA. Detection and localization of nonmetallic intraocular foreign bodies by magnetic resonance imaging. Arch Ophthalmol 1988; 106: 260-261. 